\title{
Alloplasmic introgression and DH-lines of (H. vulgare)-T. aestivum and (H. marinum ssp. gussoneanum)-T. aestivum: research models and initial material for breeding
}

\author{
Pershina L.A. ${ }^{*}$, Trubacheeva N.V. ${ }^{1}$, Osadchaya T.S. ${ }^{1}$, Kravtsova L.A. ${ }^{1}$, Belova L.I. ${ }^{1}$, \\ Belan I.A. ${ }^{2}$, Rosseeva L.P. ${ }^{2}$ \\ ${ }^{1}$ Institute of Cytology and Genetics, SB RAS, Novosibirsk, Russia \\ ${ }^{2}$ Omsk Agricultural Scientific Center, Omsk, Russia \\ *e-mail:pershina@bionet.nsc.ru
}

Introgressive hybridization is a basic method for increasing the genetic diversity of bread wheat. In our studies, genotypes of bread wheat with new intergenomic interactions by replacing the cytoplasm of wheat with an alien one are creating. These genotypes (alloplasmic lines) are using for production of introgression and homozygous introgression (DH) lines with pyramids of genes controlling resistant to fungal pathogens. Developed a set of methods aimed at restoring fertility and overcoming nuclear-cytoplasmic conflict when crossing between individual species of barley and wheat, as well as the subsequent reconstruction of nuclear genes as a result of the integration of alien genetic material into the nuclear genome of alloplasmic lines (H. vulgare) $-T$. aestivum and (H. marinum ssp. gussoneanum) $-T$. aestivum. It was shown that the peculiarities of restoring the fertility of alloplasmic lines and integration of chromosomes of barley in genome of alloplasmic lines depending on the species of barley. It was discovered that the patterns of fertility restoration of alloplasmic lines associated with the variability of nuclear and cytoplasmic genomes. It has been established that the conditions of anther culture for production of DH lines do not negatively affect nuclear-cytoplasmic compatibility in alloplasmic genotypes (H. vulgare)-T. aestivum. In our work, alloplasmic and DH lines are used as a new genotypes in breeding. DH lines serve to accelerate the selection of the desired genotypes with target genes and are included in the hybridization to obtain the initial material for breeding. Alloplasmic wheat-barley substitution and addition lines (H. marinum ssp. gussoneanum)-T. aestivum with chromosomes of $H$. marinum are used to obtain euplasmic wheat-barley introgression lines.

The positive effect of individual chromosomes of this wild barley on the content of protein in the grain was shown.

Acknowledgements: This work were supported by project No. 0324 - 2019-0039 and the RFBR grant No. 17-04-01738. 